\title{
Prevalence and selected risk factors of suicidal ideation, suicidal tendencies and suicide attempts in young people aged 13-19 years
}

\author{
Maciej Zygo ${ }^{1, A-F}$, Beata Pawłowska ${ }^{2, A, C, E-F}$, Emilia Potembska, ${ }^{3, E-F}$, Piotr Dreher, ${ }^{4,1, E-F}$, \\ Lucyna Kapka-Skrzypczak ${ }^{5,6, \mathrm{E}-\mathrm{F}}$ \\ ${ }^{1}$ M. Kaczyński Neuropsychiatric Hospital, Lublin, Poland \\ ${ }^{2}$ 2nd Department of Psychiatry and Psychiatric Rehabilitation, Medical University, Lublin, Poland \\ ${ }^{3}$ Department of Psychiatric Nursing, Medical University, Lublin, Poland \\ ${ }^{4}$ Chair and Department of Public Health, Medical University, Lublin, Poland \\ ${ }^{5}$ Department of Molecular Biology and Translational Research, Institute of Rural Health, Lublin, Poland \\ ${ }^{6}$ Department of Medical Biology and Translational Research, Faculty of Medicine, University of Information Technology \\ and Management, Rzeszow, Poland \\ A - Research concept and design, B - Collection and/or assembly of data, C - Data analysis and interpretation,
}

$D$ - Writing the article, E - Critical revision of the article, F- Final approval of article

Zygo M, Pawłowska B, Potembska E, Dreher P, Kapka-Skrzypczak L. Prevalence and selected risk factors of suicidal ideation, suicidal tendencies and suicide attempts in young people aged 13-19 years. Ann Agric Environ Med. 2019; 26(2): 329-336. doi: 10.26444/aaem/93817

\begin{abstract}
I Abstract
Objective. The aim of this study was to assess the prevalence of suicidal thoughts, tendencies and suicide attempts in young people. An attempt was also made to identify factors which, according to those young people, contributed to their suicide attempts.

Materials and method. The study involved 5,685 individuals aged 13-19 years. The participants were surveyed using an inquiry form designed by the study authors.

Results. Suicidal behaviour in adolescents correlates with the female gender, intake of psychoactive substances, running away from home, being raised in a single-parent family, addiction of family members to alcohol, and experiences of violence. Conclusions. 1) Suicidal ideation was reported by $24.66 \%$, suicidal plans - by $15.55 \%$, and suicide attempts - by $4.37 \%$ of the adolescents studied. 2) Girls were significantly more likely to attempt suicide out of a sense of helplessness, loneliness, rejection and guilt, as well as conflicts with parents and peers. Boys were significantly more likely than girls to attempt suicide as a result of pressure from peers or cyber acquaintances. 3) Suicide attempts were significantly more common among girls than boys between the ages of 13-19, and significantly more common among young people living in urban areas than those living in the countryside. 4) Significantly more young people who reported suicidal thoughts and plans and suicide attempts than those not reporting such experiences were raised in single-parent families. 5) Compared with non-suicidal controls, young people who admitted to having suicidal thoughts and plans and to having attempted suicide, were significantly more likely to report alcohol abuse by parents and experiences of psychological and physical violence from family members.
\end{abstract}

\section{Key words}

family, adolescents, suicidal behaviour

\section{INTRODUCTION}

In recent years there has been an increase in the suicide rate in Poland, both in the adult and adolescent populations [1,2, 3]. Kułaga et al. [3], who analyzed the causes of death of Polish adolescents aged 13-19 years, found that suicide was the second most common cause of death, second only to traffic accidents, and more common than drowning. In 2014, a total of 6,165 suicide attempts were fatal, which was 64 more than the previous year and 1,988 more than in 2012 [4]. In 2014, suicides were attempted by 20 individuals aged 9 or under, 71 individuals aged 10-14, and 526 individuals aged 15-19 [4]. The data collected by the General Office of Statistics [5] show that in 2016 as many as 101 young people aged 13-18

Address for correspondence: Beata Pawłowska, 2nd Department of Psychiatry and Psychiatric Rehabilitation, Medical University of Lublin, Poland, Głuska 1, 20-439 Lublin, Poland

e-mail: pawlowskabeata@tlen.pl

Received: 31.01.2018; accepted: 30.07.2018; first published: 24.08.2018 years died by suicide. A majority of those adolescents were residents of Lublin and Gdańsk [4]. Napieralska [6] argues that the suicide rate in children and adolescents aged 10-19 displays a growing tendency in both genders.

Hołyst [7] emphasizes the fact that, worldwide, suicide is among the 5 most common causes of death of adolescents aged 15-19. Suicide is the third most common cause of death in children and adolescents in the USA [8]. Steele and Doey [9] established that about $12 \%$ of American children aged 6-12 and 53\% of American adolescents aged 13-19 had suicidal thoughts. Nock et al. [10], based on a study of 6,483 young people aged $13-18$ years, showed that $12.1 \%$ of them had suicidal ideation, $4.0 \%$ had suicidal plans, and $4.1 \%$ had a history of attempted suicide. According to Zakharov et al. [11], most suicides among young people (86.5\%) are attempted by adolescents aged $14-18$ years, and $13.5 \%$ of suicides are attempted by children between 9-13 years of age. Nock et al. [10] found that the prevalence of suicidal ideation in adolescents increased rapidly between the ages of 12-17, 
while the prevalence of suicidal plans and suicide attempts increased most rapidly up to the age of 15 , and then more slowly between the ages of $15-17$ years. Nock et al. [10] found that among young people who reported suicidal ideation, $33.4 \%$ had made suicide plans and $33.9 \%$ of the latter had actually attempted suicide.

Researchers [11, 12] investigating suicidal behaviour among young people observed that girls were more likely than boys to have attempted suicide and to report suicidal ideation [10]. Similar results were obtained by Zoroglu et al. [13], who found that $13.2 \%$ of the girls and $5.4 \%$ of the boys in their study group had attempted suicide. Lewinsohn et al. [12] observed that in a sample of young people aged 12-18 years, suicide had been significantly more often attempted by girls than boys, but in 19-year-olds, the incidence of suicide attempts was similar for both genders. That study [12] indicated that in the group of girls over 13 years of age, the number of suicide attempts grew to reach a maximum between the ages of 15-18, and then decreased. The largest number of suicide attempts among boys was recorded for 15-year-olds [12]. According to Gmitrowicz [14], in a group of young people aged 13-17 years, suicide attempts were more common in boys, whereas in the group of under 13-year-olds, the incidence was higher in girls. Data also show that there is an alarming increase in suicidal attempts among Polish girls aged between 10-14 [15].

According to Swahn et al. [16], suicide attempts by adolescents are associated with early initiation of alcohol use (before the 13th birthday). Similar results were obtained by Kim and Kim [17], who found that early initiation of drinking and smoking increased the risk of suicidal ideation and suicide attempts both in girls and in boys. Wichstrøm [18] listed the following risk factors for suicidal behaviour in adolescents: female gender, alcohol abuse, being raised by a single parent, and poor self-worth. Similar observations were reported by Kelly et al. [19], who showed that alcohol abuse predisposed adolescents of both genders to suicide attempts. Borges et al. [20] found that teenagers who had attempted suicide, were significantly more likely than those who had not, to have abused alcohol and drugs. Wu and et al. [21] reported that young people who had suicidal ideation and a history of attempted suicide, were significantly more likely to have been drunk and used drugs. Moreover, adolescents who had attempted suicide were significantly more likely to have used drugs than those who reported suicidal ideation alone. Nock et al. [10] demonstrated that young people who reported suicidal thoughts and plans were significantly more likely than individuals who did not engage in such behaviour to manifest anxiety disorders, conduct disorders, and oppositional defiant disorders, and to abuse alcohol and drugs.

Blum et al. [22] observed that young people raised in single parent families were significantly more likely to report suicidal ideation and suicide attempts in comparison with their peers raised by both parents. Park et al. [23] listed parental divorce and parental alcoholism among the risk factors for suicidal thoughts in boys, as opposed to girls, for whom family factors did not increase the risk for suicidal ideation.

Another important risk factor for adolescent suicide attempts are experiences of childhood sexual [24] and physical $[25,26]$ abuse. Riggs et al. [27] established that young people who had experienced sexual abuse and physical abuse in their childhood were 3 and 5 times more likely, respectively, to have attempted suicide compared to their peers who had had no experience of these types of violence. Zoroglu et al. [13] found that compared to non-suicidal adolescents, young people who had a history of attempted suicide were significantly more likely to have experienced emotional (50.0\% vs $11.8 \%)$, physical (42.0\% vs $10.3 \%)$ and sexual $(20.3 \%$ vs $6.1 \%)$ abuse.

To summarize, data from the literature [28-33] indicate that the following factors are predictive of a suicide attempt in the adolescent population: social and demographic factors (female gender, urban background), family situation (disturbed family structure), interpersonal conflict, disturbed functioning in social roles (school difficulties, absenteeism, running away from home, difficulties in relationships with peers), substance abuse, life crises (divorce of the parents, death of a parent), psychological factors (personality traits, coping strategies), and factors related to health and biology.

\section{OBJECTIVE}

The aim of this present study was to assess the prevalence of suicidal ideation and suicide attempts in people aged 13-19 years and to determine the relationship between suicidal behaviour and selected demographic and social variables. An attempt was also made to identify factors which subjects listed as contributing to their suicide attempts.

\section{MATERIALS AND METHOD}

Participants. The study was carried out between December 2013 - April 2014. The participants were 5,685 individuals aged $13-19$ years. $70 \%(\mathrm{~N}=3,980)$ of them were girls and $30 \%$ $(\mathrm{N}=1,705)$ were boys. The participants attended schools in the Lublin Province, in Eastern Poland. 46\% $(\mathrm{N}=2,615)$ of those surveyed lived in urban areas and $54 \%(\mathrm{~N}=3,070)$ lived in rural areas. The mean age of the participants was 16.91 , median - 17. Table 1 shows socio-demographic data for the sample, by age group.

Table 1. Socio-demographics by age

\begin{tabular}{lccccc}
\hline \multirow{2}{*}{ Age in years } & $\begin{array}{c}\text { Whole } \\
\text { group }\end{array}$ & Girls & Boys & $\begin{array}{c}\text { Urban } \\
\text { residents }\end{array}$ & $\begin{array}{c}\text { Rural } \\
\text { residents }\end{array}$ \\
\cline { 2 - 6 } & $\mathrm{N}$ & $\mathrm{N}$ & $\mathrm{N}$ & $\mathrm{N}$ & $\mathrm{N}$ \\
& $(\%)$ & $(\%)$ & $(\%)$ & $(\%)$ & $(\%)$ \\
\hline \multirow{2}{*}{13} & 47 & 29 & 14 & 40 & 7 \\
& $(0.83)$ & $(0.80)$ & $(0.90)$ & $(1.58)$ & $(0.24)$ \\
\hline \multirow{2}{*}{14} & 246 & 157 & 63 & 180 & 61 \\
& $(4.33)$ & $(4.35)$ & $(4.06)$ & $(7.12)$ & $(2.09)$ \\
\hline 15 & 240 & 167 & 51 & 145 & 79 \\
& $(4.22)$ & $(4.63)$ & $(3.29)$ & $(5.73)$ & $(2.71)$ \\
\hline \multirow{2}{*}{16} & 906 & 559 & 256 & 357 & 499 \\
& $(15.94)$ & $(15.49)$ & $(16.49)$ & $(14.12)$ & $(17.09)$ \\
\hline \multirow{2}{*}{18} & 2590 & 1640 & 711 & 1133 & 1372 \\
& $(45.55)$ & $(45.45)$ & $(45.81)$ & $(44.80)$ & $(47.00)$ \\
\hline 19 & 1460 & 961 & 385 & 602 & 804 \\
& $(25.68)$ & $(26.64)$ & $(24.81)$ & $(23.80)$ & $(27.54)$ \\
\hline & 177 & 95 & 72 & 72 & 93 \\
& $(3.11)$ & $(2.63)$ & $(4.64)$ & $(2.85)$ & $(3.33)$ \\
\hline
\end{tabular}


Procedures. The survey was conducted in schools whose headmasters gave their consent to the study. An effort was made to survey the largest sample possible. Because participation in the survey was entirely voluntary, not all of the subjects completed their answer sheets. A total of 6,198 questionnaire forms were distributed, 513 of which were rejected as incomplete. All pupils willing to participate in the research filled in a questionnaire during their lessons at school; anonymity and confidentiality of data were assured.

Ethical Issues. The study was approved by the Clinical Research Ethics Board of the Medical University (No. KE0254/94/2012) and accepted by the Board of Education. On receiving the questionnaires, the participants were informed that they were entitled to receive professional help.

Measures. The participants were surveyed using a self-report questionnaire designed by the authors of the study, in which the following variables were determined: gender, age, place of residence, educational level, parents' education, structure of the participants' families (growing up in a complete, reconstructed or single-parent family), causes of the family's incompleteness (death of a parent, divorce), experiences of emotional, physical, and sexual abuse from family members and peers, self-mutilation, suicidal ideation and suicide attempts, as well as the motivation for the attempts. When designing their questionnaire, the authors attempted to include the different variables described in the literature as risk factors for suicide attempts among young people.

Statistical analysis. In order to compare the groups in terms of the selected socio-demographic variables, measured on a nominal dichotomous scale, the non-parametric chi square test was used. A p value of 0.05 was deemed statistically significant. STATISTICA 10.0 software (StatSoft, PL) was used to create the database and perform the statistical analyses.

\section{RESULTS}

The data obtained in the study showed that suicidal ideation was reported by $24.66 \%(\mathrm{~N}=1269)$, suicidal plans - by $15.55 \%$ $(\mathrm{N}=797)$, and suicide attempts - by $4.37 \%(\mathrm{~N}=225)$ of the participants.

Table 2 shows the results of a $\chi^{2}$ test, which was used to compare the numbers of boys and girls who reported suicidal ideation and suicide attempts.

Table 2. Comparison of the number of boys and girls reporting suicidal thoughts and plans and suicide attempts

\begin{tabular}{|c|c|c|c|c|c|}
\hline \multirow{2}{*}{ Suicidal behaviour } & \multicolumn{2}{|c|}{ Females } & \multicolumn{2}{|c|}{ Males } & \multirow{2}{*}{$x^{2}$} \\
\hline & $\mathrm{N}$ & $\%$ & $\mathrm{~N}$ & $\%$ & \\
\hline Suicidal thoughts & 1026 & 28.57 & 243 & 15.63 & $97.86^{* * *}$ \\
\hline Suicidal tendencies & 646 & 18.04 & 151 & 9.77 & $56.34^{* * *}$ \\
\hline Suicide attempts & 192 & 5.34 & 33 & 2.14 & $26.46^{* * *}$ \\
\hline
\end{tabular}

${ }^{*} \mathrm{p}<.05 ;{ }^{* *} \mathrm{p}<.01 ;{ }^{* * *} \mathrm{p}<.001$

Significantly more girls than boys reported having had suicidal thoughts and plans, and having attempted suicide.

Table 3 presents data regarding the age of the young people reporting suicidal thoughts and plans and suicide attempts (Tab. 3).
Table 3. Age of young people reporting suicidal thoughts, plans and suicide attempts

\begin{tabular}{|c|c|c|c|c|c|c|c|c|c|}
\hline \multicolumn{10}{|c|}{ Entire group } \\
\hline & \multicolumn{3}{|c|}{ Suicidal thoughts } & \multicolumn{3}{|c|}{ Suicidal plans } & \multicolumn{3}{|c|}{ Suicide attempts } \\
\hline Age & $\begin{array}{l}\text { Entire } \\
\text { group }\end{array}$ & $\begin{array}{l}\mathrm{Fe}- \\
\text { males }\end{array}$ & Males & $\begin{array}{l}\text { Entire } \\
\text { group }\end{array}$ & $\begin{array}{c}\mathrm{Fe}- \\
\text { males }\end{array}$ & Males & $\begin{array}{l}\text { Entire } \\
\text { group }\end{array}$ & $\begin{array}{c}\mathrm{Fe}- \\
\text { males }\end{array}$ & Males \\
\hline & $\begin{array}{c}\mathrm{N} \\
(\%)\end{array}$ & $\begin{array}{c}\mathrm{N} \\
(\%)\end{array}$ & $\begin{array}{c}\mathrm{N} \\
(\%)\end{array}$ & $\begin{array}{c}\mathrm{N} \\
(\%)\end{array}$ & $\begin{array}{c}\mathrm{N} \\
(\%)\end{array}$ & $\begin{array}{c}\mathrm{N} \\
(\%)\end{array}$ & $\begin{array}{c}\mathrm{N} \\
(\%)\end{array}$ & $\begin{array}{c}N \\
(\%)\end{array}$ & $\begin{array}{c}\mathrm{N} \\
(\%)\end{array}$ \\
\hline 13 & $\begin{array}{c}10 \\
(0.72) \\
\end{array}$ & $\begin{array}{c}8 \\
(0.78)\end{array}$ & $\begin{array}{c}2 \\
(0.82) \\
\end{array}$ & $\begin{array}{c}6 \\
(0.68)\end{array}$ & $\begin{array}{c}4 \\
(0.62) \\
\end{array}$ & $\begin{array}{c}2 \\
(1.32)\end{array}$ & $\begin{array}{c}1 \\
(0.40) \\
\end{array}$ & $\begin{array}{c}1 \\
(0.53) \\
\end{array}$ & $\begin{array}{c}0 \\
(0.00)\end{array}$ \\
\hline 14 & $\begin{array}{c}73 \\
(5.26) \\
\end{array}$ & $\begin{array}{c}54 \\
(5.29) \\
\end{array}$ & $\begin{array}{c}10 \\
(4.12) \\
\end{array}$ & $\begin{array}{c}45 \\
(5.13)\end{array}$ & $\begin{array}{c}32 \\
(4.98)\end{array}$ & $\begin{array}{c}8 \\
(5.30) \\
\end{array}$ & $\begin{array}{c}17 \\
(6.80)\end{array}$ & $\begin{array}{c}11 \\
(5.79) \\
\end{array}$ & $\begin{array}{c}4 \\
(12.12) \\
\end{array}$ \\
\hline 15 & $\begin{array}{c}67 \\
(4.82) \\
\end{array}$ & $\begin{array}{c}57 \\
(5.58)\end{array}$ & $\begin{array}{c}6 \\
(2.47) \\
\end{array}$ & $\begin{array}{c}42 \\
(4.79)\end{array}$ & $\begin{array}{c}34 \\
(5.29) \\
\end{array}$ & $\begin{array}{c}5 \\
(3.31) \\
\end{array}$ & $\begin{array}{c}14 \\
(5.60)\end{array}$ & $\begin{array}{c}12 \\
(6.32) \\
\end{array}$ & $\begin{array}{c}2 \\
(6.06) \\
\end{array}$ \\
\hline 16 & $\begin{array}{c}196 \\
(14.11)\end{array}$ & $\begin{array}{c}130 \\
(12.73) \\
\end{array}$ & $\begin{array}{c}42 \\
(17.28) \\
\end{array}$ & $\begin{array}{c}123 \\
(14.03) \\
\end{array}$ & $\begin{array}{c}77 \\
(11.98) \\
\end{array}$ & $\begin{array}{c}28 \\
(18.54) \\
\end{array}$ & $\begin{array}{c}40 \\
(16.00) \\
\end{array}$ & $\begin{array}{c}30 \\
(15.79) \\
\end{array}$ & $\begin{array}{c}3 \\
(9.09) \\
\end{array}$ \\
\hline 17 & $\begin{array}{c}616 \\
(44.35) \\
\end{array}$ & $\begin{array}{c}462 \\
(45.25) \\
\end{array}$ & $\begin{array}{c}100 \\
(41.15) \\
\end{array}$ & $\begin{array}{c}380 \\
(43.33)\end{array}$ & $\begin{array}{c}287 \\
(44.63)\end{array}$ & $\begin{array}{c}55 \\
(36.42) \\
\end{array}$ & $\begin{array}{c}101 \\
(40.40) \\
\end{array}$ & $\begin{array}{c}72 \\
(37.89) \\
\end{array}$ & $\begin{array}{c}16 \\
(48.48) \\
\end{array}$ \\
\hline 18 & $\begin{array}{c}384 \\
(27.65) \\
\end{array}$ & $\begin{array}{c}283 \\
(27.72)\end{array}$ & $\begin{array}{c}69 \\
(28.40) \\
\end{array}$ & $\begin{array}{c}251 \\
(28.62)\end{array}$ & $\begin{array}{c}191 \\
(29.70) \\
\end{array}$ & $\begin{array}{c}42 \\
(27.81) \\
\end{array}$ & $\begin{array}{c}71 \\
(28.40)\end{array}$ & $\begin{array}{c}60 \\
(31.58) \\
\end{array}$ & $\begin{array}{c}7 \\
(21.21) \\
\end{array}$ \\
\hline 19 & $\begin{array}{c}43 \\
(3.09)\end{array}$ & $\begin{array}{c}27 \\
(2.64)\end{array}$ & $\begin{array}{c}14 \\
(5.76)\end{array}$ & $\begin{array}{c}30 \\
(3.42)\end{array}$ & $\begin{array}{c}18 \\
(2.80)\end{array}$ & $\begin{array}{c}11 \\
(7.28)\end{array}$ & $\begin{array}{c}6 \\
(2.40)\end{array}$ & $\begin{array}{c}4 \\
(2.11)\end{array}$ & $\begin{array}{c}0 \\
(0.00)\end{array}$ \\
\hline
\end{tabular}

Urban

\begin{tabular}{ccccccccccc}
\hline & \multicolumn{1}{c}{ Suicidal thoughts } & \multicolumn{3}{c}{ Suicidal plans } & \multicolumn{3}{c}{ Suicide attempts } \\
\cline { 2 - 11 } & $\begin{array}{c}\text { Entire } \\
\text { Age }\end{array}$ & $\begin{array}{c}\text { Fe- } \\
\text { males }\end{array}$ & Males & $\begin{array}{c}\text { Entire } \\
\text { group }\end{array}$ & $\begin{array}{c}\text { Fe- } \\
\text { males }\end{array}$ & Males & $\begin{array}{c}\text { Entire } \\
\text { group }\end{array}$ & $\begin{array}{c}\text { Fe- } \\
\text { males }\end{array}$ & Males \\
\cline { 2 - 11 } & $\mathrm{N}$ & $\mathrm{N}$ & $\mathrm{N}$ & $\mathrm{N}$ & $\mathrm{N}$ & $\mathrm{N}$ & $\mathrm{N}$ & $\mathrm{N}$ & $\mathrm{N}$ \\
& $(\%)$ & $(\%)$ & $(\%)$ & $(\%)$ & $(\%)$ & $(\%)$ & $(\%)$ & $(\%)$ & $(\%)$ \\
\hline \multirow{2}{*}{13} & 8 & 7 & 1 & 4 & 3 & 1 & 0 & 0 & 0 \\
& $(1.25)$ & $(1.54)$ & $(0.81)$ & $(1.03)$ & $(1.11)$ & $(1.30)$ & $(0.00)$ & $(0.00)$ & $(0.00)$ \\
\hline \multirow{2}{*}{14} & 57 & 42 & 8 & 36 & 27 & 6 & 14 & 10 & 2 \\
& $(8.89)$ & $(9.25)$ & $(6.45)$ & $(9.28)$ & $(10.00)$ & $(7.79)$ & $(11.02)$ & $(10.99)$ & $(10.00)$ \\
\hline \multirow{2}{*}{15} & 40 & 35 & 4 & 25 & 21 & 3 & 10 & 9 & 1 \\
& $(6.24)$ & $(7.71)$ & $(3.23)$ & $(6.44)$ & $(7.78)$ & $(3.90)$ & $(7.87)$ & $(9.89)$ & $(5.00)$ \\
\hline \multirow{2}{*}{16} & 88 & 55 & 21 & 57 & 34 & 15 & 21 & 15 & 3 \\
& $(13.73)$ & $(12.11)$ & $(16.94)$ & $(14.69)$ & $(12,59$ & $(19.48)$ & $(16.54)$ & $(16.48)$ & $(15.00)$ \\
\hline \multirow{2}{*}{17} & 272 & 201 & 48 & 156 & 112 & 27 & 45 & 29 & 9 \\
& $(42.43)$ & $(44.27)$ & $(38.71)$ & $(40.21)$ & $(41.48)$ & $(35.06)$ & $(35.43)$ & $(31.87)$ & $(45.00)$ \\
\hline \multirow{2}{*}{18} & 155 & 100 & 36 & 96 & 64 & 20 & 35 & 26 & 5 \\
& $(24.18)$ & $(22.03)$ & $(29.03)$ & $(24.74)$ & $(23.70)$ & $(25.97)$ & $(27.56)$ & $(28.57)$ & $(25.00)$ \\
\hline \multirow{2}{*}{19} & 21 & 14 & 6 & 14 & 9 & 5 & 2 & 2 & 0 \\
& $(3.28)$ & $(3.08)$ & $(4.84)$ & $(3.61)$ & $(3.33)$ & $(6.49)$ & $(1.57)$ & $(2.20)$ & $(0.00)$ \\
\hline
\end{tabular}

Rural

\begin{tabular}{|c|c|c|c|c|c|c|c|c|c|}
\hline \multirow[b]{2}{*}{ Age } & \multicolumn{3}{|c|}{ Suicidal thoughts } & \multicolumn{3}{|c|}{ Suicidal plans } & \multicolumn{3}{|c|}{ Suicide attempts } \\
\hline & $\begin{array}{l}\text { Entire } \\
\text { group }\end{array}$ & $\begin{array}{l}\mathrm{Fe}- \\
\text { males }\end{array}$ & Males & $\begin{array}{l}\text { Entire } \\
\text { group }\end{array}$ & $\begin{array}{c}\mathrm{Fe}- \\
\text { males }\end{array}$ & Males & $\begin{array}{l}\text { Entire } \\
\text { group }\end{array}$ & $\begin{array}{c}\mathrm{Fe}- \\
\text { males }\end{array}$ & Males \\
\hline & $\begin{array}{c}N \\
(\%)\end{array}$ & $\begin{array}{c}\mathrm{N} \\
(\%)\end{array}$ & $\begin{array}{c}\mathrm{N} \\
(\%)\end{array}$ & $\begin{array}{c}\mathrm{N} \\
(\%)\end{array}$ & $\begin{array}{c}\mathrm{N} \\
(\%)\end{array}$ & $\begin{array}{c}\mathrm{N} \\
(\%)\end{array}$ & $\begin{array}{c}\mathrm{N} \\
(\%)\end{array}$ & $\begin{array}{c}N \\
(\%)\end{array}$ & $\begin{array}{c}\mathrm{N} \\
(\%)\end{array}$ \\
\hline 13 & $\begin{array}{c}2 \\
(0.29)\end{array}$ & $\begin{array}{c}1 \\
(0.19) \\
\end{array}$ & $\begin{array}{c}1 \\
(0.90) \\
\end{array}$ & $\begin{array}{c}2 \\
(0.44)\end{array}$ & $\begin{array}{c}1 \\
(0.28) \\
\end{array}$ & $\begin{array}{c}1 \\
(1.49) \\
\end{array}$ & $\begin{array}{c}1 \\
(0.87) \\
\end{array}$ & $\begin{array}{c}1 \\
(1.05)\end{array}$ & $\begin{array}{c}0 \\
(0.00)\end{array}$ \\
\hline 14 & $\begin{array}{c}15 \\
(2.15)\end{array}$ & $\begin{array}{c}11 \\
(2.04)\end{array}$ & $\begin{array}{c}2 \\
(1.80)\end{array}$ & $\begin{array}{c}9 \\
(1.99)\end{array}$ & $\begin{array}{c}5 \\
(1.42)\end{array}$ & $\begin{array}{c}2 \\
(2.99)\end{array}$ & $\begin{array}{c}3 \\
(2.61)\end{array}$ & $\begin{array}{c}1 \\
(1.05)\end{array}$ & $\begin{array}{c}2 \\
(16.67)\end{array}$ \\
\hline 15 & $\begin{array}{c}22 \\
(3.16)\end{array}$ & $\begin{array}{c}19 \\
(3.53)\end{array}$ & $\begin{array}{c}2 \\
(1.80)\end{array}$ & $\begin{array}{c}14 \\
(3.09)\end{array}$ & $\begin{array}{c}11 \\
(3.13)\end{array}$ & $\begin{array}{c}2 \\
(2.99)\end{array}$ & $\begin{array}{c}4 \\
(3.48)\end{array}$ & $\begin{array}{c}3 \\
(3.16)\end{array}$ & $\begin{array}{c}1 \\
(8.33)\end{array}$ \\
\hline 16 & $\begin{array}{c}100 \\
(14.35)\end{array}$ & $\begin{array}{c}73 \\
(13.54)\end{array}$ & $\begin{array}{c}19 \\
(17.12) \\
\end{array}$ & $\begin{array}{c}59 \\
(13.02) \\
\end{array}$ & $\begin{array}{c}42 \\
(1.93) \\
\end{array}$ & $\begin{array}{c}11 \\
(16.42) \\
\end{array}$ & $\begin{array}{c}16 \\
(13.91) \\
\end{array}$ & $\begin{array}{c}15 \\
(15.79)\end{array}$ & $\begin{array}{c}0 \\
(0.00)\end{array}$ \\
\hline 17 & $\begin{array}{c}324 \\
(46.48)\end{array}$ & $\begin{array}{c}250 \\
(46.38)\end{array}$ & $\begin{array}{c}48 \\
(43.24)\end{array}$ & $\begin{array}{c}209 \\
(46.14)\end{array}$ & $\begin{array}{c}166 \\
(47.16)\end{array}$ & $\begin{array}{c}24 \\
(35.82)\end{array}$ & $\begin{array}{c}53 \\
(46.09)\end{array}$ & $\begin{array}{c}41 \\
(43.16)\end{array}$ & $\begin{array}{c}6 \\
(50.00)\end{array}$ \\
\hline 18 & $\begin{array}{c}214 \\
(30.70) \\
\end{array}$ & $\begin{array}{c}174 \\
(32.28)\end{array}$ & $\begin{array}{c}31 \\
(27.93) \\
\end{array}$ & $\begin{array}{c}145 \\
(32.01) \\
\end{array}$ & $\begin{array}{c}119 \\
(33.81)\end{array}$ & $\begin{array}{c}21 \\
(31.34) \\
\end{array}$ & $\begin{array}{c}35 \\
(30.43) \\
\end{array}$ & $\begin{array}{c}33 \\
(34.74) \\
\end{array}$ & $\begin{array}{c}2 \\
(16.67) \\
\end{array}$ \\
\hline & $\begin{array}{c}20 \\
(2.87) \\
\end{array}$ & $\begin{array}{c}11 \\
(2.04) \\
\end{array}$ & $\begin{array}{c}8 \\
(7.21) \\
\end{array}$ & $\begin{array}{c}15 \\
(3.31)\end{array}$ & $\begin{array}{c}8 \\
(2.27) \\
\end{array}$ & $\begin{array}{c}6 \\
(8.96) \\
\end{array}$ & $\begin{array}{c}3 \\
(2.61)\end{array}$ & $\begin{array}{c}1 \\
(1.05)\end{array}$ & $\begin{array}{c}1 \\
(8.33) \\
\end{array}$ \\
\hline
\end{tabular}


The results obtained for the study sample as a whole demonstrate that the largest group of teenagers reporting suicidal thoughts, suicidal plans and suicide attempts were those aged 17 and 18.

Table 4 compares young people reporting suicidal thoughts and plans and suicide attempts with regard to their place of residence.

Table 4. Comparison of places of residence of young people reporting suicidal thoughts and plans and suicide attempts

\begin{tabular}{lllllll}
\hline \multirow{2}{*}{ Variables } & \multicolumn{3}{c}{ Urban } & \multicolumn{2}{c}{ Rural } & \multirow{2}{*}{ X $^{2}$} \\
\cline { 2 - 6 } & & $\mathrm{N}$ & $\%$ & $\mathrm{~N}$ & $\%$ & \\
\hline \multirow{3}{*}{$\begin{array}{l}\text { Entire } \\
\text { group }\end{array}$} & Suicidal thoughts & 644 & 25.50 & 698 & 24.04 & 1.55 \\
\cline { 2 - 6 } & Suicidal tendencies & 390 & 15.52 & 453 & 15.65 & 0.02 \\
\cline { 2 - 6 } & Suicide attempts & 128 & 5.07 & 115 & 3.97 & $3.80^{*}$ \\
\hline \multirow{3}{*}{ Females } & Suicidal thoughts & 457 & 29.18 & 540 & 27.89 & 0.71 \\
\cline { 2 - 6 } & Suicidal tendencies & 272 & 17.46 & 352 & 18.21 & 0.33 \\
\cline { 2 - 6 } & Suicide attempts & 92 & 5.86 & 95 & 4.90 & 1.60 \\
\hline \multirow{3}{*}{ Males } & Suicidal thoughts & 124 & 16.85 & 111 & 14.49 & 1.58 \\
\cline { 2 - 6 } & Suicidal tendencies & 77 & 10.50 & 67 & 8.80 & 1.24 \\
\cline { 2 - 6 } & Suicide attempts & 20 & 2.72 & 12 & 1.58 & 2.32 \\
\hline
\end{tabular}

${ }^{*} \mathrm{p}<.05 ;{ }^{* *} \mathrm{p}<.01 ;{ }^{* * *} \mathrm{p}<.001$

The results obtained for the whole study group suggest that suicide is attempted significantly more frequently by teenagers who live in urban areas than those who live in rural areas.
In the next stage of the study, the participants' responses to the question what had motivated them to attempt suicide were analyzed. The results obtained for the whole study sample showed that $12.69 \%(\mathrm{~N}=657)$ of the adolescents had attempted suicide out of a sense of helplessness, $8.42 \%$ $(\mathrm{N}=436)$ reported loneliness as a motive, 6.24\% $(\mathrm{N}=323)$ related their suicide attempt to rejection, 5.81\% ( N=301) had attempted suicide because they had been angry with their parents, and $5.41 \%(\mathrm{~N}=280)$ because they had been in conflict with their parents. $3.65 \%(\mathrm{~N}=189)$ of the subjects had attempted suicide because of school difficulties, $2.43 \%$ $(\mathrm{N}=126)$ because of conflicts with their peers, and $2.97 \%$ $(\mathrm{N}=154)$ out of a sense of guilt. Among the less common motives for attempting suicide, $1.49 \%(\mathrm{~N}=77)$ of the young people mentioned impulsiveness, $1.08 \%(\mathrm{~N}=56)$ - a desire for revenge, and $0.29 \%(\mathrm{~N}=15)$ - substance abuse. $0.19 \%(\mathrm{~N}=10)$ of the participants had attempted suicide having been talked into it by their friends or cyber acquaintances.

Table 5 compares the patterns of suicide attempts by girls and boys with regard to their place of residence

The data obtained in the study indicated that, in trying to commit suicide, girls were more likely than boys to have been guided by feelings of helplessness, loneliness, rejection, anger, and guilt, and conflicts with their parents and peers. Boys, on the other hand, were significantly more likely than girls to have been driven to suicide by their peers or cyber acquaintances.

When motives for suicide attempts were compared for boys and girls from urban and rural settings, it turned out that girls living in towns and cities were more likely than their countryside peers to have attempted suicide under the

Table 5. Comparison of motives for suicide attempts made by girls and boys classified by place of residence

\begin{tabular}{|c|c|c|c|c|c|c|c|c|c|}
\hline \multirow{3}{*}{ Variables } & \multicolumn{3}{|c|}{ Gender } & \multicolumn{3}{|c|}{ Urban } & \multicolumn{3}{|c|}{ Rural } \\
\hline & Females & Males & \multirow[b]{2}{*}{$x^{2}$} & Females & Males & \multirow[b]{2}{*}{$x^{2}$} & Females & Males & \multirow[b]{2}{*}{$x^{2}$} \\
\hline & $\begin{array}{c}\mathrm{N} \\
(\%)\end{array}$ & $\begin{array}{c}\mathrm{N} \\
(\%)\end{array}$ & & $\begin{array}{c}\mathrm{N} \\
(\%)\end{array}$ & $\begin{array}{c}\mathrm{N} \\
(\%)\end{array}$ & & $\begin{array}{c}\mathrm{N} \\
(\%)\end{array}$ & $\begin{array}{c}\mathrm{N} \\
(\%)\end{array}$ & \\
\hline Pressure & $\begin{array}{c}2 \\
(0.06)\end{array}$ & $\begin{array}{c}8 \\
(0.51)\end{array}$ & $11.81 * * *$ & $\begin{array}{c}1 \\
(0.06)\end{array}$ & $\begin{array}{c}3 \\
(0.41)\end{array}$ & 3.42 & $\begin{array}{c}1 \\
(0.05)\end{array}$ & $\begin{array}{c}5 \\
(0.65)\end{array}$ & $8.98^{* *}$ \\
\hline Helplessness & $\begin{array}{c}554 \\
(15.32)\end{array}$ & $\begin{array}{c}103 \\
(6.59)\end{array}$ & $74.98^{* * *}$ & $\begin{array}{c}252 \\
(16.00)\end{array}$ & $\begin{array}{c}51 \\
(6.90)\end{array}$ & $36.59 * * *$ & $\begin{array}{c}290 \\
(14.86)\end{array}$ & $\begin{array}{c}48 \\
(6.23)\end{array}$ & $37.75^{* * *}$ \\
\hline Rejection & $\begin{array}{c}266 \\
(7.36)\end{array}$ & $\begin{array}{c}57 \\
(3.65)\end{array}$ & $25.63^{* * *}$ & $\begin{array}{c}126 \\
(8.00)\end{array}$ & $\begin{array}{c}28 \\
(3.79)\end{array}$ & $1.36^{* * *}$ & $\begin{array}{c}130 \\
(6.66)\end{array}$ & $\begin{array}{c}28 \\
(3.64)\end{array}$ & $9.23^{* *}$ \\
\hline Loneliness & $\begin{array}{c}368 \\
(10.18)\end{array}$ & $\begin{array}{c}68 \\
(4.35)\end{array}$ & $47.97^{* * *}$ & $\begin{array}{c}176 \\
(11.17)\end{array}$ & $\begin{array}{c}34 \\
(4.60)\end{array}$ & $26.34 * * *$ & $\begin{array}{c}181 \\
(9.27)\end{array}$ & $\begin{array}{c}33 \\
(4.29)\end{array}$ & $18.96^{* * *}$ \\
\hline Guilt & $\begin{array}{c}127 \\
(3.51)\end{array}$ & $\begin{array}{c}27 \\
(1.73)\end{array}$ & $12.03^{* * *}$ & $\begin{array}{c}61 \\
(3.87)\end{array}$ & $\begin{array}{c}17 \\
(2.30)\end{array}$ & $3.82^{*}$ & $\begin{array}{c}63 \\
(3.23)\end{array}$ & $\begin{array}{c}9 \\
(1.17)\end{array}$ & $9.09^{* *}$ \\
\hline Psychoactive drugs & $\begin{array}{c}10 \\
(0.28)\end{array}$ & $\begin{array}{c}5 \\
(0.32)\end{array}$ & 0.07 & $\begin{array}{c}8 \\
(0.51)\end{array}$ & $\begin{array}{c}2 \\
(0.27)\end{array}$ & 0.66 & $\begin{array}{c}2 \\
(0.10)\end{array}$ & $\begin{array}{c}3 \\
(0.39)\end{array}$ & 2.48 \\
\hline Anger & $\begin{array}{c}231 \\
(6.39)\end{array}$ & $\begin{array}{c}70 \\
(4.48)\end{array}$ & $7.24^{* *}$ & $\begin{array}{c}117 \\
(7.43)\end{array}$ & $\begin{array}{c}34 \\
(4.60)\end{array}$ & $6.59^{* *}$ & $\begin{array}{c}108 \\
(5.53)\end{array}$ & $\begin{array}{c}36 \\
(4.68)\end{array}$ & 0.81 \\
\hline Revenge & $\begin{array}{c}42 \\
(1.16)\end{array}$ & $\begin{array}{c}14 \\
(0.90)\end{array}$ & 0.72 & $\begin{array}{c}23 \\
(1.46)\end{array}$ & $\begin{array}{c}5 \\
(0.68)\end{array}$ & 2.58 & $\begin{array}{c}19 \\
(0.97)\end{array}$ & $\begin{array}{c}9 \\
(1.17)\end{array}$ & 0.21 \\
\hline School problems & $\begin{array}{c}140 \\
(3.87)\end{array}$ & $\begin{array}{c}49 \\
(3.14)\end{array}$ & 1.67 & $\begin{array}{c}61 \\
(3.87)\end{array}$ & $\begin{array}{c}25 \\
(3.38)\end{array}$ & 0.34 & $\begin{array}{c}76 \\
(3.89)\end{array}$ & $\begin{array}{c}24 \\
(3.12)\end{array}$ & 0.94 \\
\hline Parent-child conflicts & $\begin{array}{c}243 \\
(6.72)\end{array}$ & $\begin{array}{c}37 \\
(2.37)\end{array}$ & $40.38^{* * *}$ & $\begin{array}{c}104 \\
(6.60)\end{array}$ & $\begin{array}{c}22 \\
(2.98)\end{array}$ & $12.85^{* * *}$ & $\begin{array}{c}132 \\
(6.76)\end{array}$ & $\begin{array}{c}14 \\
(1.82)\end{array}$ & $26.59 * * *$ \\
\hline Peer-peer conflicts & $\begin{array}{c}106 \\
(2.93)\end{array}$ & $\begin{array}{c}20 \\
(1.28)\end{array}$ & $12.52^{* * *}$ & $\begin{array}{c}50 \\
(3.17)\end{array}$ & $\begin{array}{c}10 \\
(1.35)\end{array}$ & $6.61^{* *}$ & $\begin{array}{c}50 \\
(2.56)\end{array}$ & $\begin{array}{c}9 \\
(1.17)\end{array}$ & $5.05^{*}$ \\
\hline
\end{tabular}

${ }^{*} \mathrm{p}<.05 ;{ }^{* *} \mathrm{p}<.01 ;{ }^{* * *} \mathrm{p}<.001$ 
influence of psychoactive substances ( $\mathrm{N}=8 ; 0.51 \%$ vs. $\mathrm{N}=2$; $\left.0.10 \% \chi^{2}=5.07 ; \mathrm{p}=0.02\right)$ and due to being angry with others $\left(\mathrm{N}=117 ; 7.43 \%\right.$ vs. $\left.\mathrm{N}=108 ; 5.53 \% \chi^{2}=5.25 ; \mathrm{p}=0.02\right)$. Boys who lived in the countryside did not differ significantly from their urban peers in terms of their motives for attempting suicide.

In relation to data which point to the important role of family-related factors in adolescent suicide attempts, Table 6 compares the family situation of the participants who reported and did not report suicidal ideation, suicidal tendencies and suicide attempts.

Significantly more teenagers who reported suicidal ideation and suicide attempts than those who did not report such experiences were being raised in incomplete families.
Significantly more boys who reported suicidal ideation and suicide attempts by boys from the control group were being raised by a single mother.

Alcohol abuse by the father, grandfather, and siblings was reported significantly more often by young people who admitted to having suicidal ideation than those who did not. It should be emphasized that girls who had attempted suicide were significantly more likely than non-suicidal female controls to report alcohol abuse by their parents, grandfathers, and siblings.

Table 7 compares data on experiences of parental violence in participants reporting suicidal behavior and non-suicidal controls.

Table 6. Comparison of family situation of adolescents who report and those who do not report suicidal thoughts and plans and suicide attempts

\begin{tabular}{|c|c|c|c|c|c|c|c|c|c|c|}
\hline \multirow{3}{*}{ Variables } & \multirow{3}{*}{ Gender } & \multicolumn{3}{|c|}{ Suicidal thoughts } & \multicolumn{3}{|c|}{ Suicidal plans } & \multicolumn{3}{|c|}{ Suicide attempts } \\
\hline & & NO & YES & \multirow[b]{2}{*}{$x^{2}$} & NO & YES & \multirow[b]{2}{*}{$x^{2}$} & NO & YES & \multirow[b]{2}{*}{$x^{2}$} \\
\hline & & $\begin{array}{c}\mathrm{N} \\
(\%)\end{array}$ & $\begin{array}{c}\mathrm{N} \\
(\%)\end{array}$ & & $\begin{array}{c}\mathrm{N} \\
(\%)\end{array}$ & $\begin{array}{c}\mathrm{N} \\
(\%)\end{array}$ & & $\begin{array}{c}\mathrm{N} \\
(\%)\end{array}$ & $\begin{array}{c}\mathrm{N} \\
(\%)\end{array}$ & \\
\hline \multirow{3}{*}{ Incomplete family } & Entire group & $\begin{array}{c}472 \\
(11.25)\end{array}$ & $\begin{array}{c}260 \\
(18.91)\end{array}$ & $57.09^{* * *}$ & $\begin{array}{c}551 \\
(11.77)\end{array}$ & $\begin{array}{c}182 \\
(20.99)\end{array}$ & $59.51^{* * *}$ & $\begin{array}{c}665 \\
(12.50)\end{array}$ & $\begin{array}{c}69 \\
(27.71)\end{array}$ & $66.73^{* * *}$ \\
\hline & Females & $\begin{array}{c}300 \\
(11.81)\end{array}$ & $\begin{array}{c}188 \\
(18.60)\end{array}$ & $29.38^{* * *}$ & $\begin{array}{c}354 \\
(12.19)\end{array}$ & $\begin{array}{c}134 \\
(21.04)\end{array}$ & $39.54^{* * *}$ & $\begin{array}{c}434 \\
(12.88)\end{array}$ & $\begin{array}{c}55 \\
(28.95)\end{array}$ & $49.28^{* * *}$ \\
\hline & Males & $\begin{array}{c}127 \\
(9.81)\end{array}$ & $\begin{array}{c}40 \\
(16.60)\end{array}$ & $15.70^{* * *}$ & $\begin{array}{c}144 \\
(10.46)\end{array}$ & $\begin{array}{c}25 \\
(16.78)\end{array}$ & $7.22^{*}$ & $\begin{array}{c}165 \\
(11.06)\end{array}$ & $\begin{array}{c}4 \\
(12.12)\end{array}$ & $17.30^{* * *}$ \\
\hline \multirow[b]{2}{*}{ Parental divorce } & Entire group & $\begin{array}{c}37 \\
(2.63)\end{array}$ & $\begin{array}{c}20 \\
(4.67)\end{array}$ & $4.54^{*}$ & $\begin{array}{c}44 \\
(2.81)\end{array}$ & $\begin{array}{c}13 \\
(4.91)\end{array}$ & 3.29 & $\begin{array}{c}53 \\
(3.01)\end{array}$ & $\begin{array}{c}4 \\
(5.71)\end{array}$ & 1.63 \\
\hline & Females & $\begin{array}{c}27 \\
(2.87)\end{array}$ & $\begin{array}{c}16 \\
(4.73)\end{array}$ & 2.66 & $\begin{array}{c}32 \\
(3.00)\end{array}$ & $\begin{array}{c}11 \\
(5.29)\end{array}$ & 2.81 & $\begin{array}{c}39 \\
(3.19)\end{array}$ & $\begin{array}{c}4 \\
(7.14)\end{array}$ & 2.58 \\
\hline \multirow{3}{*}{ Single mother } & Entire group & $\begin{array}{c}21 \\
(1.49)\end{array}$ & $\begin{array}{c}10 \\
(2.34)\end{array}$ & 1.40 & $\begin{array}{c}25 \\
(1.60) \\
\end{array}$ & $\begin{array}{c}6 \\
(2.26) \\
\end{array}$ & 0.60 & $\begin{array}{c}29 \\
(1.65) \\
\end{array}$ & $\begin{array}{c}2 \\
(2.86) \\
\end{array}$ & 0.59 \\
\hline & Females & $\begin{array}{c}16 \\
(1.70)\end{array}$ & $\begin{array}{c}6 \\
(1.78)\end{array}$ & 0.01 & $\begin{array}{c}19 \\
(1.78)\end{array}$ & $\begin{array}{c}3 \\
(1.44)\end{array}$ & 0.12 & $\begin{array}{c}21 \\
(1.72)\end{array}$ & $\begin{array}{c}1 \\
(1.79)\end{array}$ & 0.01 \\
\hline & Males & $\begin{array}{c}4 \\
(1.10) \\
\end{array}$ & $\begin{array}{c}4 \\
(6.78) \\
\end{array}$ & $8.76^{* *}$ & $\begin{array}{c}5 \\
(1.32) \\
\end{array}$ & $\begin{array}{c}3 \\
(7.69) \\
\end{array}$ & $7.68^{* *}$ & $\begin{array}{c}7 \\
(1.71) \\
\end{array}$ & $\begin{array}{c}1 \\
(12.50) \\
\end{array}$ & $4.87^{*}$ \\
\hline \multirow{3}{*}{$\begin{array}{l}\text { Alcohol abuse by } \\
\text { the mother }\end{array}$} & Entire group & $\begin{array}{c}34 \\
(0.97)\end{array}$ & $\begin{array}{c}32 \\
(2.71)\end{array}$ & $19.19^{* * *}$ & $\begin{array}{c}40 \\
(1.02) \\
\end{array}$ & $\begin{array}{c}26 \\
(3.50)\end{array}$ & $27.53^{* * *}$ & $\begin{array}{c}51 \\
(1.14) \\
\end{array}$ & $\begin{array}{c}14 \\
(6.54) \\
\end{array}$ & $43.43^{* * *}$ \\
\hline & Females & $\begin{array}{c}26 \\
(1.20) \\
\end{array}$ & $\begin{array}{c}27 \\
(3.07)\end{array}$ & $12.88^{* * *}$ & $\begin{array}{c}31 \\
(1.25)\end{array}$ & $\begin{array}{c}22 \\
(3.99) \\
\end{array}$ & $19.78^{* * *}$ & $\begin{array}{c}40 \\
(1.39)\end{array}$ & $\begin{array}{c}12 \\
(7.23)\end{array}$ & $31.98^{* * *}$ \\
\hline & Males & $\begin{array}{c}7 \\
(0.68)\end{array}$ & $\begin{array}{c}3 \\
(1.51)\end{array}$ & 1.41 & $\begin{array}{c}7 \\
(0.64)\end{array}$ & $\begin{array}{c}3 \\
(2.42)\end{array}$ & $4.33^{*}$ & $\begin{array}{c}9 \\
(0.75)\end{array}$ & $\begin{array}{c}1 \\
(4.00)\end{array}$ & 3.17 \\
\hline \multirow{2}{*}{$\begin{array}{l}\text { Alcohol abuse by } \\
\text { the father }\end{array}$} & Entire group & $\begin{array}{c}336 \\
(9.58) \\
\end{array}$ & $\begin{array}{c}261 \\
(22.06) \\
\end{array}$ & $123.97^{* * *}$ & $\begin{array}{c}424 \\
(10.80)\end{array}$ & $\begin{array}{c}174 \\
(23.39) \\
\end{array}$ & $88.70^{* * *}$ & $\begin{array}{c}532 \\
(11.91) \\
\end{array}$ & $\begin{array}{c}66 \\
(30.84) \\
\end{array}$ & $65.65^{* * *}$ \\
\hline & Males & $\begin{array}{c}82 \\
(7.98)\end{array}$ & $\begin{array}{c}33 \\
(16.50)\end{array}$ & $14.29^{* * *}$ & $\begin{array}{c}98 \\
(8.96)\end{array}$ & $\begin{array}{c}18 \\
(14.40)\end{array}$ & $3.86^{*}$ & $\begin{array}{c}111 \\
(9.30)\end{array}$ & $\begin{array}{c}2 \\
(20.00)\end{array}$ & 3.26 \\
\hline \multirow{3}{*}{$\begin{array}{l}\text { Alcohol abuse by } \\
\text { the grandfather }\end{array}$} & Entire group & $\begin{array}{c}170 \\
(4.85)\end{array}$ & $\begin{array}{c}120 \\
(10.14) \\
\end{array}$ & $42.74^{* * *}$ & $\begin{array}{c}202 \\
(5.15) \\
\end{array}$ & $\begin{array}{c}86 \\
(11.56) \\
\end{array}$ & $44.44^{* * *}$ & $\begin{array}{c}263 \\
(5.89) \\
\end{array}$ & $\begin{array}{c}25 \\
(11.68) \\
\end{array}$ & $11.87^{* * *}$ \\
\hline & Females & $\begin{array}{c}102 \\
(4.69)\end{array}$ & $\begin{array}{c}89 \\
(10.11)\end{array}$ & $31.45^{* * *}$ & $\begin{array}{c}128 \\
(5.14)\end{array}$ & $\begin{array}{c}61 \\
(11.03)\end{array}$ & $26.95^{* * *}$ & $\begin{array}{c}169 \\
(5.85)\end{array}$ & $\begin{array}{c}20 \\
(12.05)\end{array}$ & $10.39^{* * *}$ \\
\hline & Males & $\begin{array}{c}50 \\
(4.87) \\
\end{array}$ & $\begin{array}{c}20 \\
(10.05) \\
\end{array}$ & $8.31^{* *}$ & $\begin{array}{c}55 \\
(5.03) \\
\end{array}$ & $\begin{array}{c}15 \\
(12.10)\end{array}$ & $10.28^{* * *}$ & $\begin{array}{c}68 \\
(5.70)\end{array}$ & $\begin{array}{c}2 \\
(8.00)\end{array}$ & 0.24 \\
\hline \multirow{3}{*}{$\begin{array}{l}\text { Alcohol abuse by } \\
\text { siblings }\end{array}$} & Entire group & $\begin{array}{c}29 \\
(0.83)\end{array}$ & $\begin{array}{c}25 \\
(2.11)\end{array}$ & $12.85^{* * *}$ & $\begin{array}{c}33 \\
(0.84)\end{array}$ & $\begin{array}{c}22 \\
(2.96)\end{array}$ & $24.06^{* * * *}$ & $\begin{array}{c}46 \\
(1.03)\end{array}$ & $\begin{array}{c}8 \\
(3.74)\end{array}$ & $13.13^{* * *}$ \\
\hline & Females & $\begin{array}{c}15 \\
(0.69)\end{array}$ & $\begin{array}{c}12 \\
(1.37)\end{array}$ & 3.26 & $\begin{array}{c}17 \\
(0.68)\end{array}$ & $\begin{array}{c}11 \\
(1.99)\end{array}$ & $8.50^{* *}$ & $\begin{array}{c}23 \\
(0.80)\end{array}$ & $\begin{array}{c}5 \\
(3.01)\end{array}$ & $8.48^{* *}$ \\
\hline & Males & $\begin{array}{c}10 \\
(0.97)\end{array}$ & $\begin{array}{c}9 \\
(4.50)\end{array}$ & $13.65^{* * *}$ & $\begin{array}{c}10 \\
(0.91)\end{array}$ & $\begin{array}{c}9 \\
(7.20)\end{array}$ & $28.89 * * *$ & $\begin{array}{c}16 \\
(1.34)\end{array}$ & $\begin{array}{c}2 \\
(8.00)\end{array}$ & $7.47^{* *}$ \\
\hline
\end{tabular}

${ }^{*} \mathrm{p}<.05 ;{ }^{* *} \mathrm{p}<.01 ;{ }^{* * *} \mathrm{p}<.001$ 
Table 7. Comparison of the numbers of suicidal and non-suicidal young people who reported and did not report experiences of violence

\begin{tabular}{|c|c|c|c|c|c|c|c|c|c|c|}
\hline \multirow{3}{*}{ Violence } & \multirow{3}{*}{ Gender } & \multicolumn{3}{|c|}{ Suicidal thoughts } & \multicolumn{3}{|c|}{ Suicidal plans } & \multicolumn{3}{|c|}{ Suicide attempts } \\
\hline & & NO & YES & & NO & YES & & NO & YES & \\
\hline & & $\begin{array}{c}\mathrm{N} \\
(\%)\end{array}$ & $\begin{array}{c}\mathrm{N} \\
(\%)\end{array}$ & $x^{2}$ & $\begin{array}{c}\mathrm{N} \\
(\%)\end{array}$ & $\begin{array}{c}\mathrm{N} \\
(\%)\end{array}$ & $x^{2}$ & $\begin{array}{c}\mathrm{N} \\
(\%)\end{array}$ & $\begin{array}{c}\mathrm{N} \\
(\%)\end{array}$ & $x^{2}$ \\
\hline \multirow{3}{*}{ Psychological violence } & Entire group & $\begin{array}{c}358 \\
(19.88)\end{array}$ & $\begin{array}{c}415 \\
(47.32)\end{array}$ & $216.32^{* * *}$ & $\begin{array}{c}484 \\
(23.11)\end{array}$ & $\begin{array}{c}292 \\
(50.34)\end{array}$ & $163.51^{* * *}$ & $\begin{array}{c}669 \\
(26.73)\end{array}$ & $\begin{array}{c}105 \\
(60.69)\end{array}$ & $90.81^{* * *}$ \\
\hline & Females & $\begin{array}{c}215 \\
(19.98)\end{array}$ & $\begin{array}{c}299 \\
(47.09)\end{array}$ & $139.60^{* * *}$ & $\begin{array}{c}305 \\
(23.70)\end{array}$ & $\begin{array}{c}209 \\
(49.64)\end{array}$ & $101.51^{* * *}$ & $\begin{array}{c}428 \\
(27.16)\end{array}$ & $\begin{array}{c}85 \\
(62.50)\end{array}$ & $74.52^{* * *}$ \\
\hline & Males & $\begin{array}{c}111 \\
(19.96)\end{array}$ & $\begin{array}{c}82 \\
(49.40)\end{array}$ & $56.54^{* * *}$ & $\begin{array}{c}140 \\
(22.76)\end{array}$ & $\begin{array}{c}56 \\
(52.34)\end{array}$ & $40.30^{* * *}$ & $\begin{array}{c}185 \\
(26.35)\end{array}$ & $\begin{array}{c}10 \\
(52.63)\end{array}$ & $6.47^{* *}$ \\
\hline \multirow[b]{2}{*}{ Physical violence } & Entire group & $\begin{array}{c}249 \\
(13.85)\end{array}$ & $\begin{array}{c}264 \\
(30.14)\end{array}$ & $100.79^{* * *}$ & $\begin{array}{c}311 \\
(14.88)\end{array}$ & $\begin{array}{c}201 \\
(34.72)\end{array}$ & $115.05^{* * *}$ & $\begin{array}{c}436 \\
(17.45)\end{array}$ & $\begin{array}{c}75 \\
(43.35)\end{array}$ & $70.20^{* * *}$ \\
\hline & Females & $\begin{array}{c}78 \\
(7.26)\end{array}$ & $\begin{array}{c}160 \\
(25.24)\end{array}$ & $107.39 * * *$ & $\begin{array}{c}109 \\
(8.49)\end{array}$ & $\begin{array}{c}127 \\
(30.24)\end{array}$ & $125.47^{* * *}$ & $\begin{array}{c}181 \\
(11.51)\end{array}$ & $\begin{array}{c}55 \\
(40.44)\end{array}$ & $88.05^{* * *}$ \\
\hline \multirow{3}{*}{ Sexual violence } & Entire group & $\begin{array}{c}18 \\
(1.00)\end{array}$ & $\begin{array}{c}32 \\
(3.65)\end{array}$ & $22.55^{* * *}$ & $\begin{array}{c}21 \\
(1.00)\end{array}$ & $\begin{array}{c}29 \\
(5.01)\end{array}$ & $39.54^{* * *}$ & $\begin{array}{c}34 \\
(1.36)\end{array}$ & $\begin{array}{c}16 \\
(9.25)\end{array}$ & $54.80^{* * *}$ \\
\hline & Females & $\begin{array}{c}11 \\
(1.03)\end{array}$ & $\begin{array}{c}24 \\
(3.79)\end{array}$ & $15.10^{* * *}$ & $\begin{array}{c}12 \\
(0.94)\end{array}$ & $\begin{array}{c}23 \\
(5.48)\end{array}$ & $32.41^{* * *}$ & $\begin{array}{c}21 \\
(1.34)\end{array}$ & $\begin{array}{c}14 \\
(10.29)\end{array}$ & $50.00^{* * *}$ \\
\hline & Males & $\begin{array}{c}5 \\
(0.90)\end{array}$ & $\begin{array}{c}4 \\
(2.41) \\
\end{array}$ & 2.37 & $\begin{array}{c}5 \\
(0.81)\end{array}$ & $\begin{array}{c}4 \\
(3.74)\end{array}$ & 6.34 & $\begin{array}{c}8 \\
(1.14)\end{array}$ & $\begin{array}{c}1 \\
(5.26) \\
\end{array}$ & 2.55 \\
\hline \multirow{3}{*}{ Family violence } & Entire group & $\begin{array}{c}155 \\
(8.63)\end{array}$ & $\begin{array}{c}241 \\
(27.48)\end{array}$ & $166.07^{* * *}$ & $\begin{array}{c}224 \\
(10.72)\end{array}$ & $\begin{array}{c}173 \\
(29.83)\end{array}$ & $130.98^{* * *}$ & $\begin{array}{c}325 \\
(13.01)\end{array}$ & $\begin{array}{c}73 \\
(42.20)\end{array}$ & $108.76^{* * *}$ \\
\hline & Females & $\begin{array}{c}88 \\
(8.22) \\
\end{array}$ & $\begin{array}{c}184 \\
(28.93)\end{array}$ & $127.82^{* * *}$ & $\begin{array}{c}135 \\
(10.53)\end{array}$ & $\begin{array}{c}137 \\
(32.46)\end{array}$ & $113.87^{* * *}$ & $\begin{array}{c}213 \\
(13.55)\end{array}$ & $\begin{array}{c}59 \\
(43.38) \\
\end{array}$ & $83.20^{* * *}$ \\
\hline & Males & $\begin{array}{c}52 \\
(9.34)\end{array}$ & $\begin{array}{c}42 \\
(25.45)\end{array}$ & $29.20^{* * *}$ & $\begin{array}{c}71 \\
(11.53)\end{array}$ & $\begin{array}{c}24 \\
(22.64)\end{array}$ & $9.78^{* * *}$ & $\begin{array}{c}89 \\
(12.68)\end{array}$ & $\begin{array}{c}7 \\
(36.84)\end{array}$ & $9.36^{* *}$ \\
\hline
\end{tabular}

${ }^{*} \mathrm{p}<.05 ;{ }^{* *} \mathrm{p}<.01 ;{ }^{* * *} \mathrm{p}<.001$

The results obtained for the entire study sample and separately for girls and boys show that individuals who had experienced psychological and physical violence in the family were significantly more likely to report suicidal ideation, suicidal tendencies and suicide attempts than those who did not report such behaviour. Also, girls who reported suicidal behaviour were significantly more likely than girls in the control group to have experienced sexual abuse. Experiences of psychological violence were reported by more than $60 \%$ of suicidal girls, and experiences of physical violence by $68 \%$ of boys who had attempted suicide. In the group of participants who had attempted suicide, $43 \%$ of girls and $37 \%$ of boys had had experiences of family violence.

\section{DISCUSSION}

The results obtained in this study show that in the surveyed group of young people aged 13-19 years, the prevalence of suicidal thoughts was $25 \%$, suicidal plans $-16 \%$, and nonfatal suicide attempts $-4.4 \%$. These results are consistent with the results obtained by Nock et al. [10], who found that in their study group consisting of 6,483 young people aged $13-18$ years, $4.1 \%$ of the respondents had attempted suicide. In the presented study, the greatest incidence of suicidal behaviours was observed in 17-year-olds. These results correspond with the opinion of Zakharov et al. [11], who believe that most young people attempt suicide at the ages of $14-18$, and Lewinsohn et al. [12], who have reported that the number of fatal suicide attempts increases in the group of girls aged over 13, to reach a maximum between the ages of 15-18, and then slowly decrease. The presented study, similar to studies by other authors [10-13], also showed that girls were significantly more likely than boys to report suicidal thoughts and plans and suicide attempts.

Statistics show that suicide deaths are more common among men than women [4], and studies conducted in the group of 13-18-year-olds show that more girls than boys make non-fatal suicide attempts. The results of the current study are consistent with the observations made by Makara et al. [15], who have drawn attention to the significant increase in the number of suicides among Polish girls aged between 10-14. Gmitrowicz et al. [1] found that girls were almost 4 times more likely to be admitted to a psychiatric ward because of suicide attempts than were boys. Similar conclusions were formulated by Mroczkowska-Juchkiewicz et al. [32], who emphasized that suicide attempts were more common among city girls.

The results of the current study show that suicide is attempted more often by young people residi9ng in urban areas than those living in the countryside. This finding is in contrast to a study by Borges et al. [20], who did not observe any statistically significant differences in terms of place of residence between young people who have and those who have not attempted suicide. In contrast to the results of the current study, Makara et al. [15] observed that in the Lublin Province there have been more suicide attempts among rural residents.

In the current study it was also found that girls from urban areas were more likely to have attempted suicide under the 
influence of psychoactive substances. Bedford et al. [16] point out that early alcohol initiation is associated with suicide attempts among young people, and Kim and Kim [17] believe that early initiation of alcohol use increases the risk of suicidal thoughts and suicide attempts in both girls and boys. Wichstrøm [18] and Kelly et al. [19] count intoxication with alcohol and harmful alcohol use among factors that predispose young people to suicide. Wu et al. [21] and Nock et al. [10] point out that young people who report suicidal behaviour are significantly more likely to get intoxicated with alcohol and use drugs than their peers who do not report suicidal behaviour.

Analysis of the data from the current study regarding young people's motives for attempting suicide demonstrated that girls were significantly more likely than boys to attempt suicide out of a sense of helplessness, loneliness, rejection and guilt, as well as due to conflicts with their parents and peers. Boys were significantly more likely than girls to be driven to suicide by their peers or Internet acquaintances. These results correspond with the findings of Gmitrowicz et al. [1], who showed that the motives for suicide attempts among adolescents included a sense of meaninglessness in life, loneliness, lack of support from friends and family, inability to solve difficult situations, and impulsiveness.

The obtained results for the current study allowed formulation of the hypotheses that environmental and family factors play an important role in suicide behaviour among adolescents. Analysis of the data showed that young people, both girls and boys, who reported suicidal behaviour were significantly more likely than their non-suicidal peers to come from an incomplete family. This observation corresponds with Wichstrøm's [18] finding that being brought up in a single-parent family (and alcohol abuse) are predictive of adolescent suicide attempts. Blum et al. [22] ascertained that young people raised in single-parent families were significantly more likely to report suicidal ideation and suicide attempts in comparison to their peers who had both parents. In this presented study, boys who reported suicidal behaviour were more likely to be raised by a single mother than were their non-suicidal peers. This finding is consistent with the opinion of Park et al. [23], who count parental divorce and alcohol abuse by parents among risk factors for suicidal ideation in boys. It should be emphasized that the results of the current study show that alcohol abuse by the father, grandfather, and siblings is reported significantly more often by girls and boys who admit to suicidal thoughts and plans than those who do not admit to suicidal ideation. At the same time, significantly more girls who have attempted suicide than non-suicidal female controls report alcohol abuse by their mothers, grandfathers and siblings. This is in line with the opinion of Mroczkowska-Juchkiewicz et al. [32], who believe that suicide attempts by young people are associated with family problems and parental alcoholism.

The presented results are consistent with the views of researchers who list experiences of sexual [24] and physical abuse $[25,26]$ as risk factors for suicide attempts among adolescents. The young people who reported suicidal thoughts and plans and suicide attempts were significantly more likely than control subjects to have experienced psychological and physical violence from family members and peers. Additionally, girls who reported suicidal behaviour were also more likely to have had experiences of sexual violence. Riggs et al. [27] emphasize that young people who have experienced sexual abuse and physical abuse in their childhood are 3 and 5 times more likely, respectively, to attempt suicide compared to their peers who have had no experience of these types of violence. Zoroglu et al. [13] found that compared to nonsuicidal adolescents, young people with a history of attempted suicide are significantly more likely to have experienced emotional (50.0\% vs $11.8 \%)$, physical (42.0\% vs $10.3 \%)$ and sexual $(20.3 \%$ vs $6.1 \%)$ abuse.

The presented study on the prevalence of suicidal behaviour and factors contributing to such behaviour among young people fully corresponds with the National Mental Health Programme. The analysis of the risk factors related to suicide attempts by adolescents is crucial to clinicians and professionals who design preventive programs.

\section{CONCLUSIONS}

1) Suicidal ideation was reported by $24.66 \%$, suicidal plans by $15.55 \%$, and suicide attempts by $4.37 \%$ of $13-9$-year-old participants.

2) Girls were significantly more likely than boys to have attempted suicide out of a sense of helplessness, loneliness, rejection and guilt, as well as due to conflicts with their parents and peers. Boys were significantly more likely than girls to have been driven to suicide by their peers or cyber acquaintances.

3) Suicide attempts were significantly more common among girls than boys and among young people living in urban areas than those living in the countryside.

4) Young people who reported suicidal behaviour were significantly more likely than those not reporting such experiences to be brought up in single-parent families.

5) Compared with the non-suicidal controls, young people who reported suicidal ideation and had a history of attempted suicide were significantly more likely to report alcohol abuse by parents and experiences of psychological and physical violence from family members.

Limitations. The study has severallimitations: 1) the survey was conducted only in those schools whose headmasters had given their consent to the study; 2) part of the students did not agree to take part in the survey, despite its anonymous character; 3) part of the students' parents did not agree to their children participating in the study. Moreover, 513 questionnaires were rejected on the grounds of being incomplete. Girls were more willing than boys to participate, which is why the female group was much larger than the male group. Because of these limitations, the results should be interpreted with caution, and the epidemiological data concerning the prevalence of suicidal ideation and suicide attempts in the described group of adolescents may be underestimated.

\section{REFERENCES}

1. Gmitrowicz A, Wolanek U, Madej A, Makara-Studzińska M. [Motives for suicide attempts by youth aged 13-19]. J Education Health Sport. 2015; 5(6): 51-64. In Polish.

2. Gmitrowicz A. [Social and psychiatric determinants of suicidal attempts in adolescents]. Post Psychiatr Neurol. 1999; 8: 457-64. In Polish.

3. Kułaga Z, Litwin M, Wójcik P, Jakubowska-Winecka A, Grajda A, Gurzkowska B, et al. Aktualne trendy zewnętrznych przyczyn zgonów dzieci i młodzieży w Polsce. Probl Hig Epidemiol. 2009; 90(3): 332-341. 
4. General Police Headquarters. [Suicide]. Available from: http:// statystyka.policja.pl (access: 2017.11.15) In Polish.

5. General Office of Statistics. [Poland is catching up with Japan as regards the suicide rate. Ed. Bankier.pl]. Available from: http://www.bankier. $\mathrm{pl}$ (access: 2017.11.15) In Polish.

6. Napieralska E. [Epidemiology of child and adolescents deaths by suicide in Poland during the years 1999-2006]. Probl Hig Epidemiol. 2010; 91(1): 92-98. In Polish.

7. Hołyst B. [Motivation for adolescent suicide. Ministry of Justice. Probation]. 2012; 3: 19-42. In Polish.

8. Centers for Disease Control, 2012. Web-based Injury Statistics Query and Reporting System TM Search. Centers for Disease Control and Prevention, National Center for Injury Prevention and Control, Atlanta, GA. Available from: http://www.cdc. gov/injury/wisqars/index.html (access: 2017.11.15).

9. Steele MM, Doey T. Suicidal behaviour in children and adolescents. Part 1: Etiology and risk factors. Can J Psychiatry. 2007; 52(6 Suppl 1): 21S-33S.

10. Nock MK, Green JG, Hwang I, McLaughlin KA, Sampson NA, Zaslavsly $\mathrm{AM}$, et al. Prevalence, correlates, and treatment of lifetime suicidal behavior among adolescents: Results from the National Comorbidity Survey Replication Adolescent Supplement. JAMA Psychiatry. 2013; 70(3): 300-310.

11. Zakharov S, Navratil T, Pelclova D. Suicide attempts by deliberate selfpoisoning in children and adolescents. Psychiatry Res. 2013; 210(1): 302-307.

12. Lewinsohn PM, Rohde P, Seeley JR, Baldwin CL. Gender differences in suicide attempts from adolescence to young adulthood. J Am Acad Child Adolesc Psychiatry. 2001; 40(4): 427-434.

13. Zoroglu SS, Tuzun U, Sar V, Tutkun U, Savaçs HA, Ozturk M, et al. Suicide attempts and self-mutilation among Turkish high school students in relation with abuse, neglect and dissociation. Psychiatry Clin Neurosci. 2003; 57(1): 119-126.

14. Gmitrowicz A. [Determinants of adolescent suicide]. Suicydologia. 2005; 1: 71-77. In Polish.

15. Makara-Studzińska M, Sygit K, Sygit M, Gozdziewska M, Zubilewicz J, Krys-Noszczyk K. Analysis of the phenomenon of attempted suicides in 1978-2010 in Poland, with particular emphasis on rural areas of Lublin Province. Ann Agric Environ Med. 2012; 19(4): 762-769.

16. Swahn MH, Bossarte RM, Sullivent III EE. Age of Alcohol Use Initiation, Suicidal Behavior, and Peer and Dating Violence Victimization and Perpetration Among High-Risk, Seventh-Grade Adolescents. Pediatrics. 2008; 121(2): 297-305.

17. Kim DS, Kim HS. Early Initiation of Alcohol Drinking, Cigarette Smoking, and Sexual Intercourse Linked to Suicidal Ideation and Attempts: Findings from the 2006 Korean Youth Risk Behavior Survey. Yonsei Med J. 2010; 51(1): 18-26.
18. Wichstrøm L. Predictors of adolescent suicide attempts: A nationally representative longitudinal study of Norwegian adolescents. J Am Acad Child Adolesc Psychiatry. 2000; 39(5): 603-610.

19. Kelly TM, Lynch KG, Donovan JE, Clark DB. Alcohol use disorders and risk factor interactions for adolescent suicidal ideation and attempts. Suicide Life Threat Behav. 2001; 31(2): 181-193.

20. Borges G, Walters EE, Kessler RC. Associations of substance use, abuse, and dependence with subsequent suicidal behavior. Am J Epidemiol. 2000; 151(8): 781-789.

21. Wu P, Hoven CW, Liu X, Cohen P, Fuller CJ, Shaffer D. Substance use, suicidal ideation and attempts in children and adolescents. Suicide Life Threat Behav. 2004; 34(4): 408-420.

22. Blum RW, Beuhring T, Shew ML, Bearinger LH, Sieving RE, Resnick MD. The effects of race/ethnicity, income, and family structure on adolescent risk behaviors. Am J Public Health. 2000; 90(12): 1879-1884.

23. Park HS, Schepp KG, Jang EH, Koo HY. Predictors of suicidal ideation among high school students by gender in South Korea. J Sch Health. 2006; 76(5): 181-188.

24. Brown J, Cohen P, Johnson JG, Smailes EM. Childhood abuse and neglect: Specificity of effects on adolescent and young adult depression and suicidality. J Am Acad Child Adolesc Psychiatry. 1999; 38(12): 1490-1496.

25. McHolm AE, MacMillan, Jamieson E. The relationship between childhood physical abuse and suicidality among depressed women: Results from a community sample. Am J Psychiatry. 2003; 160(5): 933-938.

26. Salzinger S, Rosario M, Feldman RS, Ng-Mak DS. Adolescent suicidal behavior: Associations with preadolescent physical abuse and selected risk and protective factors. J Am Acad Child Adolesc Psychiatry. 2007; 46(7): 859-866.

27. Riggs S, Alario AJ, McHorney C. Health risk behaviors and attempted suicide in adolescents who report prior maltreatment. J Pediatr. 1990; 116(5): 815-821.

28. Rosa K. [Adolescent suicide attempts. Sociological characteristics.]. Przegl Lek. 2007; 1: 24-30. In Polish.

29. Rosa K. [Suicidal attempts. Sociological characteristics]. Łódź: Oficyna Wydawnicza Instytutu Medycyny Pracy; 1996. In Polish.

30. Rosa K, Gmitrowicz A. [Dynamics of change of social demographic and psychological factors in adolescents after suicidal attempts]. Post Psych Neurol. 1999; 8: 465-470. In Polish.

31. Rosa K, Gmitrowicz A. [Regional Program for the Prevention of Suicide Among Adolescents, Part I. Characteristics of the Łodź region population]. Suicydologia. 2007; 3(1): 18-22. In Polish.

32. Mroczkowska-Juchkiewicz A, Krawiec P, Pawłowska-Kamieniak A, Gołyska D, Kominek K, Pac-Kożuchowska E. Intentional poisonings in urban and rural children - a 6-year retrospective single centre study. Ann Agric Environ Med. 2016; 23(3): 482-486.

33. Cash SJ, Bridge JA. Epidemiology of Youth Suicide and Suicidal Behavior. Curr Opin Pediatr. 2009; 21(5): 613-619. 